\title{
The emergence of an ethical duty to disclose genetic research results: international perspectives
}

\author{
Bartha Maria Knoppers, Yann Joly, Jacques Simard and Francine Durocher
}

European Journal of Human Genetics (2006) 14, 1322. doi:10.1038/sj.ejhg.5201747

Correction to: European Journal of Human Genetics (2006) 14, 1170-1178. doi:10.1038/sj.ejhg.5201690

Due to a typesetting error the second paragraph of the Conclusion section of the above paper contained an error; the corrected paragraph is shown below.

However handled, the issue of notifying (or not) participants of results should be disclosed and agreed to in advance (ie on the consent form). As stated by the European Commission in 2004:

Public trust in research surrounding genetic testing is largely dependent on how the use of samples and data in and from biobanks is undertaken and communicated. This applies in particular to the communication of study results and, where appropriate, of individual test results. 